\title{
On the similarity between Dirichlet-Neumann with Interface Artificial Compressibility and Robin-Neumann schemes for the solution of fluid-structure interaction problems
}

\author{
Joris Degroote ${ }^{\mathrm{a}}$ \\ ${ }^{a}$ Department of Flow, Heat and Combustion Mechanics, Ghent University, Sint-Pietersnieuwstraat 41, B-9000 Ghent, \\ Belgium
}

Keywords: fluid-structure interaction, partitioned, Robin-Neumann, Dirichlet-Neumann, Interface Artificial Compressibility, finite volume

\section{Introduction}

In this note, the similarity between two implicit, partitioned solution techniques for fluidstructure interaction (FSI) problems is analyzed using finite volume discretization of the flow equations. Fluid-structure interaction refers to the mutual interaction between a fluid flow and a flexible structure. Partitioned solution techniques solve the flow equations and the structural equations separately. These techniques are classified as implicit (or strongly coupled) if they satisfy the interaction conditions on the fluid-structure interface in each time step and as explicit (or loosely coupled) if they do not.

Both techniques analyzed in this note use block Gauss-Seidel (GS) iterations, meaning that the flow equations and the structural equations are solved consecutively within a time step until some convergence tolerance is reached. As the flow and structural equations are solved separately, the interaction conditions on the fluid-structure interface have to be converted into boundary conditions on the common boundary of the fluid and structure subdomains. Several types of boundary conditions can be applied, resulting in different decompositions. In the case of Dirichlet-Neumann (DN) decomposition, the flow equations are solved with a Dirichlet boundary condition (given velocity) on the fluid-structure interface, while the structural equations are solved with a Neumann boundary condition (given stress) on the interface. Conversely, RobinNeumann (RN) decomposition, introduced in [1], denotes a Robin boundary condition on the fluid side of the interface and a Neumann boundary condition on the structure side.

The first technique in this comparison is block Gauss-Seidel iterations applied to the monolithic system previously multiplied by a suitable permutation matrix, leading to a Robin-Neumann decomposition (GS-RN). This first technique includes a simplified version of the structural model in the flow equations by means of a Robin boundary condition to accelerate the convergence of the GS iterations [1, 2]. The second technique is block Gauss-Seidel iterations with DirichletNeumann decomposition and Interface Artificial Compressibility (GS-DN-IAC). This second

Email address: Joris. Degroote@UGent . be (Joris Degroote)

URL: http: / /www. fsi.ugent. be/(Joris Degroote)

Preprint submitted to Journal of Computational Physics

April 11, 2011 
technique includes a local, linearized version of the structural model in the flow equations by means of pressure-dependent source terms in the fluid cells adjacent to the fluid-structure interface. Source terms were added to both the continuity equation and the momentum equations in [3], whereas only the continuity equation was modified in [4-6]. It is important to mention that the density is constant in the IAC method, despite the name of this method which was given due to the similarity with artificial compressibility schemes to solve flow problems [7]. In [8], Artificial Compressibility was applied to the entire fluid domain and not only in the cells adjacent to the fluid-structure interface.

\section{Governing equations}

The fluid $(f)$ and structure $(s)$ subdomains are indicated as $\Omega_{f}$ and $\Omega_{s}$ and their boundaries as $\Gamma_{f}$ and $\Gamma_{s}$. The fluid-structure interface $\Gamma_{f s}=\Gamma_{f} \cap \Gamma_{s}$ is the common boundary of these subdomains, as indicated in Figure 1. The governing equations are immediately given in timediscrete form, using backward Euler discretization for simplicity. The notation $\delta_{t}$ is defined as

$$
\delta_{t} z^{n+1}=\frac{z^{n+1}-z^{n}}{\Delta t}
$$

for any time-dependent variable $z$, with the superscript $n$ denoting the time step and $\Delta t$ the time step size.

The flow equations for the incompressible fluid with density $\rho_{f}$ in $\Omega_{f}^{n+1}$ are given by

$$
\begin{gathered}
\nabla \cdot \boldsymbol{v}^{n+1}=0 \\
\delta_{t} \boldsymbol{v}^{n+1}+\nabla \cdot \boldsymbol{v}^{n+1}\left(\boldsymbol{v}^{n+1}-\boldsymbol{w}^{n+1}\right)-\frac{1}{\rho_{f}} \nabla \cdot \boldsymbol{T}_{f}^{n+1}=\mathbf{0}
\end{gathered}
$$

in arbitrary Lagrangian-Eulerian (ALE) formulation, with $\boldsymbol{v}$ the fluid velocity and $\boldsymbol{w}$ the grid velocity. The interface position is treated implicitly. Body forces are omitted for simplicity. For a Newtonian fluid with dynamic viscosity $\mu$, the Cauchy stress tensor $\boldsymbol{T}_{f}$ is defined as

$$
\boldsymbol{T}_{f}=-p \boldsymbol{I}+2 \mu \boldsymbol{G}
$$

with $p$ the pressure and

$$
\boldsymbol{G}=\frac{1}{2}\left(\nabla \boldsymbol{v}+(\nabla \boldsymbol{v})^{\mathrm{T}}\right)
$$

the strain rate tensor. The structure in $\Omega_{s}^{n+1}$ is governed by

$$
\rho_{s} \delta_{t t} \boldsymbol{u}^{n+1}-\nabla \cdot \boldsymbol{T}_{s}^{n+1}=\mathbf{0}
$$

in Lagrangian formulation, with $\boldsymbol{u}$ the displacement. The relation between the Cauchy stress tensor $\boldsymbol{T}_{s}$ and the strain tensor is given by the constitutive law of the material. On the interface $\Gamma_{f_{s}}^{n+1}$, the kinematic equilibrium

$$
\boldsymbol{v}^{n+1}=\delta_{t} \boldsymbol{u}^{n+1}
$$

and the dynamic equilibrium

$$
\boldsymbol{T}_{f}^{n+1} \cdot \boldsymbol{n}^{n+1}=\boldsymbol{T}_{s}^{n+1} \cdot \boldsymbol{n}^{n+1}
$$


need to be satisfied, with $\boldsymbol{n}^{n+1}$ the normal pointing outwards of $\Omega_{f}^{n+1}$. Moreover, the normal velocity of the fluid grid has to match the normal structural velocity on $\Gamma_{f s}^{n+1}$.

$$
\boldsymbol{w}^{n+1} \cdot \boldsymbol{n}^{n+1}=\delta_{t} \boldsymbol{u}^{n+1} \cdot \boldsymbol{n}^{n+1}
$$

For simplicity, interpolation on the fluid-structure interface is disregarded in the above equations.

\section{Comparison of GS-RN and GS-DN-IAC}

The GS technique performs coupling iterations between the flow solver and the structural solver within each time step, as can be seen in Algorithm 1. The difference between GS-RN and GS-DN lies in the boundary conditions that are applied during the solution of the flow equations (line 3). In the following comparison, it is assumed that coupling iterations are performed until the convergence criteria for the equilibrium conditions on the fluid-structure interface are satisfied.

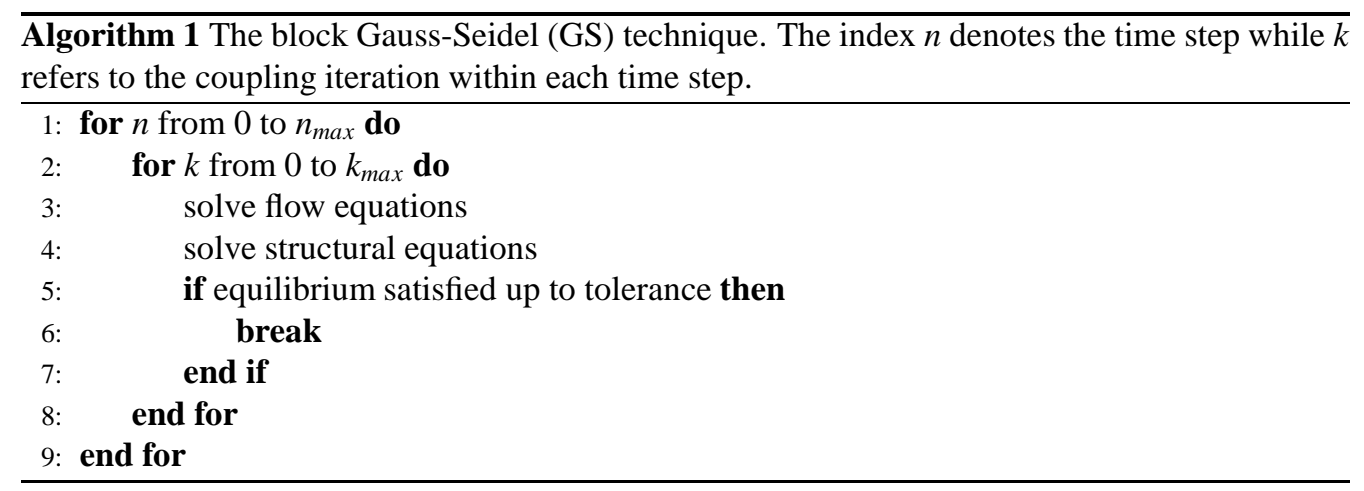

\subsection{Block Gauss-Seidel iterations with Robin-Neumann decomposition (GS-RN)}

When solving the flow equations, GS-RN uses a Robin boundary condition on $\Gamma_{f s}^{k+1}$ for the fluid, given by

$$
\boldsymbol{v}^{k+1}+\alpha \boldsymbol{T}_{f}^{k+1} \cdot \boldsymbol{n}^{k}=\delta_{t} \boldsymbol{u}^{k}+\alpha \boldsymbol{T}_{s}^{k} \cdot \boldsymbol{n}^{k}
$$

with $\alpha$ a suitable function of the fluid-structure interface. The superscript $k+1$ indicates the current coupling iteration in the current time step $(n+1)$. It will be explained in Section 4 how the coefficient $\alpha$ can be determined. For the grid velocity, a Dirichlet boundary condition

$$
\boldsymbol{w}^{k} \cdot \boldsymbol{n}^{k}=\delta_{t} \boldsymbol{u}^{k} \cdot \boldsymbol{n}^{k}
$$

is applied. Consequently, the fluid domain deforms in each coupling iteration. In Eq. (7a) and Eq. (7b), the values of $\boldsymbol{u}, \boldsymbol{T}_{s}$ and $\boldsymbol{n}$ are determined by the structural calculation at the end coupling iteration $k$.

While solving the structural equations, the most recent flow values are used in the Neumann boundary condition

$$
\boldsymbol{T}_{s}^{k+1} \cdot \boldsymbol{n}^{k+1}=\boldsymbol{T}_{f}^{k+1} \cdot \boldsymbol{n}^{k+1} .
$$


To analyze the similarities between GS-RN and GS-DN-IAC, the finite volume discretization of Eqs. (2) will be assumed, giving for cell $i$

$$
\begin{gathered}
\frac{V_{i}^{k}-V_{i}^{n}}{\Delta t}+\sum_{j}\left(\boldsymbol{v}_{i, j}^{k+1}-\boldsymbol{w}_{i, j}^{k}\right) \cdot \boldsymbol{n}_{i, j}^{k} S_{i, j}^{k}=0 \\
\frac{\boldsymbol{v}_{i}^{k+1} V_{i}^{k}-\boldsymbol{v}_{i}^{n} V_{i}^{n}}{\Delta t}+\sum_{j} \boldsymbol{v}_{i, j}^{k+1}\left(\boldsymbol{v}_{i, j}^{k+1}-\boldsymbol{w}_{i, j}^{k}\right) \cdot \boldsymbol{n}_{i, j}^{k} S_{i, j}^{k}-\text { Stress }=\mathbf{0}
\end{gathered}
$$

with $V_{i}$ the cell volume, $S_{i, j}$ the area of face $j$ and $\boldsymbol{n}_{i, j}$ the normal pointing outwards of face $j$. The vectors $\boldsymbol{v}_{i, j}$ and $\boldsymbol{w}_{i, j}$ denote respectively the fluid velocity and the grid velocity on face $j$ of cell $i$. Again, the geometrical values (including $V_{i}, S_{i, j}$ and $\boldsymbol{n}_{i, j}$ ) correspond with the structural calculation in the previous coupling iteration. The discretization of the last term in Eq. (2b) is not relevant for the remainder of the comparison so this is not specified.

Considering the Robin condition Eq. (7a), the factor $\left(\boldsymbol{v}_{i, j}^{k+1}-\boldsymbol{w}_{i, j}^{k}\right) \cdot \boldsymbol{n}_{i, j}^{k}$ for face $j=m$ on $\Gamma_{f s}^{k+1}$ becomes

$$
\left(\boldsymbol{v}_{i, m}^{k+1}-\boldsymbol{w}_{i, m}^{k}\right) \cdot \boldsymbol{n}_{i, m}^{k}=\left(\delta_{t} \boldsymbol{u}_{i, m}^{k}+\alpha_{i, m}\left(\boldsymbol{T}_{s, i, m}^{k}-\boldsymbol{T}_{f, i, m}^{k+1}\right) \cdot \boldsymbol{n}_{i, m}^{k}-\boldsymbol{w}_{i, m}^{k}\right) \cdot \boldsymbol{n}_{i, m}^{k} .
$$

Substitution of Eq. (7b) and Eq. (7c) leads to

$$
\left(\boldsymbol{v}_{i, m}^{k+1}-\boldsymbol{w}_{i, m}^{k}\right) \cdot \boldsymbol{n}_{i, m}^{k}=\alpha_{i, m} \boldsymbol{n}_{i, m}^{k} \cdot\left(\boldsymbol{T}_{f, i, m}^{k}-\boldsymbol{T}_{f, i, m}^{k+1}\right) \cdot \boldsymbol{n}_{i, m}^{k} .
$$

The summations in Eqs. (8) are subsequently split into a term corresponding with face $j=m$ on $\Gamma_{f s}^{k+1}$ and the terms corresponding with the other faces $j \neq m$ not on $\Gamma_{f s}^{k+1}$, giving

$$
\begin{aligned}
\frac{V_{i}^{k}-V_{i}^{n}}{\Delta t} & +\sum_{j \neq m}\left(\boldsymbol{v}_{i, j}^{k+1}-\boldsymbol{w}_{i, j}^{k}\right) \cdot \boldsymbol{n}_{i, j}^{k} S_{i, j}^{k} \\
& =-\alpha_{i, m} \boldsymbol{n}_{i, m}^{k} \cdot\left(\boldsymbol{T}_{f, i, m}^{k}-\boldsymbol{T}_{f, i, m}^{k+1}\right) \cdot \boldsymbol{n}_{i, m}^{k} S_{i, m}^{k} \\
\frac{\boldsymbol{v}_{i}^{k+1} V_{i}^{k}-\boldsymbol{v}_{i}^{n} V_{i}^{n}}{\Delta t} & +\sum_{j \neq m} \boldsymbol{v}_{i, j}^{k+1}\left(\boldsymbol{v}_{i, j}^{k+1}-\boldsymbol{w}_{i, j}^{k}\right) \cdot \boldsymbol{n}_{i, j}^{k} S_{i, j}^{k}-\text { Stress } \\
& =-\alpha_{i, m} \boldsymbol{v}_{i, m}^{k+1} \boldsymbol{n}_{i, m}^{k} \cdot\left(\boldsymbol{T}_{f, i, m}^{k}-\boldsymbol{T}_{f, i, m}^{k+1}\right) \cdot \boldsymbol{n}_{i, m}^{k} S_{i, m}^{k} .
\end{aligned}
$$

3.2. Block Gauss-Seidel iterations with Dirichlet-Neumann decomposition and Interface Artificial Compressibility (GS-DN-IAC) by

When solving the flow equations, GS-DN uses a Dirichlet boundary condition on $\Gamma_{f s}^{k+1}$, given

$$
\boldsymbol{v}^{k+1}=\delta_{t} \boldsymbol{u}^{k}
$$

The boundary conditions for the grid velocity and for the structural equations are identical to those in Section 3.1. The IAC then adds the source terms

$$
\begin{gathered}
-\frac{p_{i, m}^{k+1}-p_{i, m}^{k}}{\Delta t} \frac{\mathrm{d}\left(\boldsymbol{u}_{i, m} \cdot \boldsymbol{n}_{i, m}\right)}{\mathrm{d} p_{i, m}} S_{i, m}^{k} \\
-\boldsymbol{v}_{i}^{k+1} \frac{p_{i, m}^{k+1}-p_{i, m}^{k}}{\Delta t} \frac{\mathrm{d}\left(\boldsymbol{u}_{i, m} \cdot \boldsymbol{n}_{i, m}\right)}{\mathrm{d} p_{i, m}} S_{i, m}^{k} \\
4
\end{gathered}
$$


to the right-hand sides of Eqs. (8), respectively, but only in cells adjacent to the fluid-structure interface. The coefficient $\mathrm{d}\left(\boldsymbol{u}_{i, m} \cdot \boldsymbol{n}_{i, m}\right) / \mathrm{d} p_{i, m}$ is similar to $\alpha$. Its value is determined a priori and calculated as explained in Section 4. Together with the first terms of Eqs. (8), these source terms become a linear approximation for $\left(V_{i}^{k+1}-V_{i}^{n}\right) / \Delta t$ and $\left(v_{i}^{k+1} V_{i}^{k+1}-v_{i}^{n} V_{i}^{n}\right) / \Delta t[6]$.

Combination of interface conditions for the fluid velocity (Eq. (7b)) and the grid velocity (Eq. (11)) yields

$$
\boldsymbol{w}^{k} \cdot \boldsymbol{n}^{k}=\delta_{t} \boldsymbol{u}^{k} \cdot \boldsymbol{n}^{k}=\boldsymbol{v}^{k+1} \cdot \boldsymbol{n}^{k} .
$$

As a result, the second term in the continuity equation vanishes on the face that lies on the fluidstructure interface, giving

$$
\begin{aligned}
\frac{V_{i}^{k}-V_{i}^{n}}{\Delta t} & +\sum_{j \neq m}\left(\boldsymbol{v}_{i, j}^{k+1}-\boldsymbol{w}_{i, j}^{k}\right) \cdot \boldsymbol{n}_{i, j}^{k} S_{i, j}^{k} \\
& =-\frac{p_{i, m}^{k+1}-p_{i, m}^{k}}{\Delta t} \frac{\mathrm{d}\left(\boldsymbol{u}_{i, m} \cdot \boldsymbol{n}_{i, m}\right)}{\mathrm{d} p_{i, m}} S_{i, m}^{k} \\
\frac{\boldsymbol{v}_{i}^{k+1} V_{i}^{k}-\boldsymbol{v}_{i}^{n} V_{i}^{n}}{\Delta t} & +\sum_{j \neq m} \boldsymbol{v}_{i, j}^{k+1}\left(\boldsymbol{v}_{i, j}^{k+1}-\boldsymbol{w}_{i, j}^{k}\right) \cdot \boldsymbol{n}_{i, j}^{k} S_{i, j}^{k}-\text { Stress } \\
& =-\boldsymbol{v}_{i}^{k+1} \frac{p_{i, m}^{k+1}-p_{i, m}^{k}}{\Delta t} \frac{\mathrm{d}\left(\boldsymbol{u}_{i, m} \cdot \boldsymbol{n}_{i, m}\right)}{\mathrm{d} p_{i, m}} S_{i, m}^{k} .
\end{aligned}
$$

\subsection{Discussion}

On the fluid-structure interface, the pressure is usually much larger than the viscous stresses. If $\boldsymbol{T}_{f}$ is simplified to $-p \boldsymbol{I}$, Eqs. (10) become

$$
\begin{gathered}
\frac{V_{i}^{k}-V_{i}^{n}}{\Delta t}+\sum_{j \neq m}\left(\boldsymbol{v}_{i, j}^{k+1}-\boldsymbol{w}_{i, j}^{k}\right) \cdot \boldsymbol{n}_{i, j}^{k} S_{i, j}^{k} \\
=-\alpha_{i, m}\left(p_{i, m}^{k+1}-p_{i, m}^{k}\right) S_{i, m}^{k} \\
\frac{\boldsymbol{v}_{i}^{k+1} V_{i}^{k}-\boldsymbol{v}_{i}^{n} V_{i}^{n}}{\Delta t}+\sum_{j \neq m} \boldsymbol{v}_{i, j}^{k+1}\left(\boldsymbol{v}_{i, j}^{k+1}-\boldsymbol{w}_{i, j}^{k}\right) \cdot \boldsymbol{n}_{i, j}^{k} S_{i, j}^{k}-\text { Stress } \\
=-\alpha_{i, m} \boldsymbol{v}_{i, m}^{k+1}\left(p_{i, m}^{k+1}-p_{i, m}^{k}\right) S_{i, m}^{k} .
\end{gathered}
$$

Hence, under the assumption of small viscous stresses on the interface, Eq. (14a) and Eq. (15a) are equal if

$$
\alpha_{i, m}=\frac{1}{\Delta t} \frac{\mathrm{d}\left(\boldsymbol{u}_{i, m} \cdot \boldsymbol{n}_{i, m}\right)}{\mathrm{d} p_{i, m}} .
$$

With that value of $\alpha_{i, m}$, the difference between Eq. (14b) and Eq. (15b) is that the right-hand side of the former contains the cell velocity $\boldsymbol{v}_{i}^{k+1}$ while the right-hand side of the latter contains the face velocity $v_{i, m}^{k+1}$. This difference, however, vanishes as the grid size tends to zero.

As could already be seen from Eq. (7a), the coefficient $\alpha_{i, m}$ relates a change in velocity of the interface to a change in stress on the interface. If the parameter $\alpha_{i, m}\left(\operatorname{resp} . \mathrm{d}\left(\boldsymbol{u}_{i, m} \cdot \boldsymbol{n}_{i, m}\right) / \mathrm{d} p_{i, m}\right)$ is set so that it approximates the actual velocity/stress (resp. displacement/pressure) relation of the structural model, then an approximation for the structural model is included into the flow calculation. This implies that both GS-RN and GS-DN-IAC take the fluid-structure interaction into account while solving the flow equations, as opposed to GS-DN. 


\section{Remarks}

For the flow in a straight flexible tube, Fourier stability analysis has demonstrated that only the source term in the continuity equation is required for fast convergence of the GS-DN-IAC iterations as long as the fluid velocity is lower than the wave speed [3]. Hence, only the source term of Eq. (12a) is added to the continuity equation in [4-6].

In [1], an analytical expression for the coefficient $\alpha$ is obtained by considering a membrane so that the structural equations can be written in the same form as the Robin boundary condition. Moreover, an optimal value for $\alpha$ derived from a Fourier analysis has been proposed in [9]. Conversely, the structural equations are solved twice in [6], each time with a different pressure on the interface, followed by a finite difference approximation of $\mathrm{d}\left(\boldsymbol{u}_{i, m} \cdot \boldsymbol{n}_{i, m}\right) / \mathrm{d} p_{i, m}$. Both approaches are valuable. The choice of the technique to calculate the coefficients is independent of the choice between GS-RN and GS-DN-IAC.

Both GS-RN and GS-DN-IAC use a relation between the displacement of a point on the interface and the pressure in that same point. This is not a good approximation if the pressure at that point causes a displacement of the entire structure. Therefore, both techniques are suitable for a tube where the radius of a tube segment is mainly determined by the pressure in that segment and less suitable for a cantilever beam where a pressure difference between the top and the bottom near the free end bends the entire beam.

\section{Conclusions}

GS-RN and GS-DN-IAC are two different implementations of the same concept, namely including a local, linear approximation for the structural behaviour into the flow equations. To include this linearized structural model, GS-DN-IAC violates the mass conservation in the fluid cells adjacent to the fluid-structure interface during the coupling iterations (but not in the result) while GS-RN uses a boundary condition. Additionally, GS-DN-IAC neglects the viscous stresses on the fluid-structure interface in this linearized structural model (but not in the result).

\section{Acknowledgments}

J. Degroote gratefully acknowledges a postdoctoral fellowship of the Research Foundation Flanders (FWO).

\section{References}

[1] S. Badia, F. Nobile, C. Vergara, Fluid-structure partitioned procedures based on Robin transmission conditions, Journal of Computational Physics 227 (14) (2008) 7027-7051.

[2] F. Nobile, C. Vergara, An effective fluid-structure interaction formulation for vascular dynamics by generalized Robin conditions, SIAM Journal on Scientific Computing 30 (2) (2008) 731-763.

[3] J. Degroote, P. Bruggeman, R. Haelterman, J. Vierendeels, Stability of a coupling technique for partitioned solvers in FSI applications, Computers \& Structures 86 (23-24) (2008) 2224-2234.

[4] K. Riemslagh, J. Vierendeels, E. Dick, Coupling of a Navier-Stokes solver and an elastic boundary solver for unsteady problems, in: K. Papailiou, D. Tsahalis, J. Périaux, C. Hirsch, M. Pandolfi (Eds.), $2^{\text {nd }}$ European Computational Fluid Dynamics Conference, John Wiley \& Sons, Athens, Greece, 1040-1045, 1998.

[5] K. Riemslagh, J. Vierendeels, E. Dick, An efficient coupling procedure for flexible wall fluid-structure interaction, in: 3th European Congress on Computational Methods in Applied Sciences and Engineering, Barcelona, Spain, 13, 2000 . 


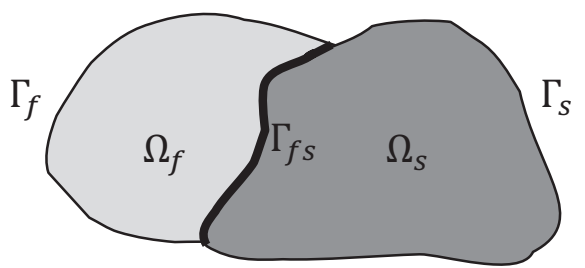

Figure 1: The fluid subdomain $\Omega_{f}$, the solid subdomain $\Omega_{s}$, their boundaries $\Gamma_{f}$ and $\Gamma_{s}$ and the fluid-structure interface $\Gamma_{f s}$.

[6] J. Degroote, A. Swillens, P. Bruggeman, R. Haelterman, P. Segers, J. Vierendeels, Simulation of fluid-structure interaction with the interface artificial compressibility method, Communications in Numerical Methods in Engineering 26 (3) (2010) 276-289.

[7] C. Merkle, M. Athavale, Time-accurate unsteady incompressible flow algorithm based on artificial compressibility, in: $8^{\text {th }}$ AIAA Computational Fluid Dynamics Conference, Honolulu, HI, USA, 397-407, aIAA 1987-1137, 1987.

[8] P. Råback, E. Järvinen, J. Ruokolainen, Computing the Artificial Compressibility Field for Partitioned FluidStructure Interaction Simulations, in: $8^{\text {th }}$ World Congress on Computational Mechanics, $5^{\text {th }}$ European Congress on Computational Methods in Applied Sciences and Engineering, Venice, Italy, 1, 2008.

[9] L. Gerardo-Giorda, F. Nobile, C. Vergara, Analysis and optimization of Robin-Robin partitioned procedures in fluidstructure interaction problems, SIAM Journal on Numerical Analysis 48 (6) (2010) 2091-2116. 